This document is the Accepted Manuscript version of a Published Work that appeared in final form in Journal of Physical Chemistry B, copyright (c) American Chemical society after peer review and technical editing by the publisher. To access the final edited and published work see https://pubs.acs.org/doi/abs/10.1021/acs.jpcb.7b00990

\title{
Lateral Pressure Profile and Free Volume Properties in Phospholipid Membranes Containing Anesthetics
}

\author{
Balázs Fábián, ${ }^{1,2}$ Marcello Sega ${ }^{3}$, Vladimir P. Voloshin,, ${ }^{4,5}$ Nikolai N. \\ Medvedev, ${ }^{4,5}$ and Pál Jedlovszky ${ }^{6,7,8^{*}}$
}

${ }^{1}$ Department of Inorganic and Analytical Chemistry, Budapest University of Technology and Economics, Szt. Gellért tér 4, H-1111 Budapest, Hungary

${ }^{2}$ Institut UTINAM (CNRS UMR 6213), Université Bourgogne Franche-Comté, 16 route de Gray, F-25030 Besançon, France

${ }^{3}$ Faculty of Physics, University of Vienna, Sensengasse 8/9, A-1090 Vienna, Austria

${ }^{4}$ Novosibirsk State University, Novosibirsk, Russia

${ }^{5}$ Institute of Chemical Kinetics and Combustion, SB RAS, Novosibirsk, Russia

${ }^{6}$ Department of Chemistry, Eszterházy Károly University, Leányka utca 6, H3300 Eger, Hungary

${ }^{7}$ MTA-BME Research Group of Technical Analytical Chemistry, Szt. Gellért tér 4, H-1111 Budapest, Hungary

${ }^{8}$ Laboratory of Interfaces and Nanosize Systems, Institute of Chemistry, Eötvös Loránd University, Pázmány P. Stny 1/A, H-1117 Budapest, Hungary

Running title: Pressure profile in membranes

*Electronic mail: jedlovszky.pal@uni-eszterhazy.hu 


\section{ABSTRACT}

The effect of four general anesthetics, namely chloroform, halothane, diethyl ether, and enflurane on the free volume fraction and lateral pressure profiles in a fully hydrated dipalmitoylphosphatidylcholime (DPPC) membrane is investigated by means of computer simulation. In order to find changes that can be related to the molecular mechanism of anesthesia as well as its pressure reversal, the simulations are performed both at atmospheric and high (1000 bar) pressures. The obtained results show that the additional free volume occurring in the membrane is localized around the anesthetic molecules themselves. Correspondingly, the fraction of the free volume is increased in the outer of the two membrane regions (i.e., at the outer edge of the hydrocarbon phase) where anesthetic molecules prefer to stay in every case. As a consequence, the presence of anesthetics decreases the lateral pressure in the nearby region of the lipid chain ester groups, in which the anesthetic molecules themselves do not penetrate. Both of these changes, occurring upon introducing anesthetics in the membrane are clearly reverted by the increase of the global pressure. These findings are in accordance both with the more than sixty years old "critical volume hypothesis" of Mullins, and with the more recent "lateral pressure hypothesis" of Cantor. Our results suggest that if anesthesia is indeed caused by conformational changes of certain membrane-bound proteins, induced by changes in the lateral pressure profile, as proposed by Cantor, the relevant conformational changes are expected to occur in the membrane region where the ester groups are located. 


\section{INTRODUCTION}

In spite of the fact that general anesthetics are routinely used in the everyday surgical practice for more than one and a half centuries, and that it is also known for more than a century that they act in the membrane of certain cells, still very little is known about the molecular mechanism of general anesthesia. Some hypotheses assume that anesthesia is caused by the specific interaction between the anesthetic molecules and certain membranebound proteins. ${ }^{1-5}$ However, in the frame of such "protein theories" it is very difficult to account for the large chemical variety of the general anesthetics. Further, neither the protein(s), nor their active site(s) responsible for the anesthetic action have been unambiguously identified yet.

Alternative explanations, called "lipid theories" conjecture that anesthetics alter certain properties of the lipid matrix, which, in turn, alters the conformation, and hence also the function of certain proteins. ${ }^{6-13}$ Several such properties of the lipid membrane have been hypothesized to be behind the molecular mechanism of anesthesia in the past six decades. Thus, in his "critical volume hypothesis", Mullins claimed that anesthesia occurs if the molar volume of the membrane exceeds a certain limit. ${ }^{6}$ This assumption was later rationalized by claiming that anesthetics increase the ordering of the lipid tails, which leads to an increased thickness of the membrane. ${ }^{11,12,14}$ In some other studies the fluidity of the membrane was thought to be the relevant property in this respect. ${ }^{15,16}$ Although a number of experimental ${ }^{11,12,15,16}$ and computer simulation studies ${ }^{17-20}$ provided results that are consistent with the above hypotheses, other studies found these properties to be insensitive to the presence of anesthetics, ${ }^{21-25}$ or even provided results contradicting some of these assumptions. $^{26-29}$ 
This controversy of the existing results largely originates from the fact that different anesthetics as well as membranes of different compositions have been considered in the different studies. Nevertheless, any possible explanation of the molecular mechanism of anesthesia in the frame of the lipid theories has to be valid for all general anesthetics as well as for all reasonable models of the cell membrane. Further, as it is well known since the 1950 s, the anesthetic effect is reverted at elevated pressures (i.e., at about 100-200 bar). ${ }^{15,30-32}$ Thus, any such explanation has also to account for this pressure reversal.

In 1997 Cantor suggested that the relevant membrane property lying behind the molecular mechanism of anesthesia might be the lateral pressure profile. ${ }^{13}$ According to this suggestion, anesthetics modify this profile in such a way that it changes the conformational equilibrium and hence also the function of certain proteins. Although this hypothesis became rather popular in the past years, its experimental test seems to be almost impossible, as it would require the measurement of certain components of the pressure tensor with an Angström resolution. Computer simulation investigation of this hypothesis, on the other hand, is hindered by the fact that it requires the local expression of a quantity (i.e., the pressure, more precisely, its lateral component) that is inherently non-local. According to the mechanical definition, the pressure contribution coming from the interaction of a particle pair can be calculated as a line integral over an open path connecting the two particles, but the resulting pressure depends, in general, on the choice of the path. ${ }^{33}$ However, it was shown that certain reasonable choices of the integration path, such as the Irwing-Kirkwood ${ }^{34}$ and the Harasima ${ }^{35}$ profiles provide compatible results with each other at least for the lateral component of the pressure. ${ }^{36}$ The use of the Harasima path has two additional advantages. First, this way the lateral pressure contribution of such a pair interaction can be distributed between the positions of the two interacting particles, which makes the calculation of the lateral pressure profile computationally very efficient. ${ }^{37}$ Further, unlike the Irwing-Kirkwood 
contour, the Harasima path can also be used in cases when the potential energy of the system is not pairwise additive. ${ }^{36}$ The importance of this feature lies in the fact that even if the potential functions used in the simulation are pairwise additive, in cases when the long range part of the electrostatic interaction is taken into account by means of the Ewald summation method $^{38-40}$ or any of its mesh variants, ${ }^{41-43}$ the reciprocal space term of this correction makes the total potential energy of the system not pairwise additive.

Although it was shown by Sonne et al. more than a decade ago how the lateral pressure contribution of the Ewald summation can be taken into account, ${ }^{36}$ its large computational cost prevents its use in simulations of systems as large as needed in studying lipid membranes. This difficulty led to the common practice in calculating lateral pressure profiles in membranes that the system is simulated using one of the fast, particle mesh variants of the Ewald method, and the pressure profile is calculated a posteriori on a large set of saved sample configurations, replacing the Ewald method with cut-off electrostatics using a cut-off value as large as possible. ${ }^{44-52}$ However, such a change in the potential between the simulation and the subsequent analysis might lead to a surprisingly large systematic error in the calculated pressure values, which can add up to several hundred bars if the applied cut-off is not sufficiently large. ${ }^{53,54}$ Very recently we demonstrated how lateral pressure profile can be calculated on the fly, in an accurate and computationally very efficient way using the Harasima path, when the long range part of the electrostatic interaction is taken into account in the simulation by one of the fast particle mesh Ewald methods. ${ }^{53}$ The capability of this method for such calculations was later demonstrated by calculating the lateral pressure profile at the liquid-vapor interface of several molecular liquids. ${ }^{55}$

In our recent publications we have studied a fully hydrated dipalmitoylphosphatidylcholine (DPPC) bilayer, as a simple model of the cell membrane, in the absence of anesthetics as well as in the presence of four widely used general anesthetic 
molecules, namely chloroform, halothane, diethyl ether, and enflurane, both at atmospheric pressure and at 1000 bar, considering both the gel $(\beta)^{56}$ and the biologically more relevant liquid crystalline $(\alpha)$ phase $^{57}$ of the bilayer. Our main approach was based on the idea that relevant membrane properties related to anesthesia must change in the same way upon addition of any general anesthetics, and in the opposite way upon increasing the pressure. Although a number of the membrane properties (e.g., density profiles, tail and headgroup orientation, tail ordering, etc.) were tested, we found that only the lateral membrane density (or area per headgroup) meets the above two criteria. The finding that in systems where anesthesia is supposed to occur the area per headgroup value exceeds a certain threshold is also consistent with the old critical volume hypothesis of Mullins, ${ }^{6}$ although it rationalizes this hypothesis in a different way than what was thought for a long time, namely, through the increase of the area rather than the thickness of the membrane.

Among the membrane properties investigated so far, the lateral pressure profile is missing, due to reason related to the technical difficulties discussed above. Having now at hand an efficient tool to calculate the local pressure, including also long-range interactions, ${ }^{51}$ enables us to calculate the lateral pressure profile also in lipid membranes in a computationally feasible way. Therefore, we have repeated our simulations, performed at $310 \mathrm{~K}$, corresponding to the biologically more relevant $\alpha$ phase of the bilayer, by calculating also the pressure profile in the membrane. To further investigate the origin of the observed effect of the anesthetics and overall pressure on the lateral density, we have performed a detailed analysis of the free volume properties of the membrane, using the Voronoi-Delaunay method, ${ }^{58-61}$ both in the presence and absence of anesthetics as well as both at atmospheric and high pressure. Again, we are looking for changes that occur in the same direction upon addition of any of the anesthetics considered, but in the opposite direction upon increasing the pressure. 
This paper is organized as follows. In section 2 details of the computations, including the molecular dynamics simulations performed, the calculation of the lateral pressure profile, and Voronoi-Delaunay analysis of the free volume properties are given. The obtained results are presented and detailed in section 3. Finally, in section 4 the most important conclusions of this study are discussed and summarized.

\section{COMPUTATIONAL METHODS}

2.1. Molecular Dynamics Simulations. Simulations of the fully hydrated neat DPPC bilayer as well as bilayers containing four different general anesthetics, namely chloroform (CF), halothane (HAL), diethyl ether (DE), and enflurane (ENF) have been performed on the isothermal-isobaric $(N, p, T)$ ensemble at the pressures of 1 and 1000 bar. The schematic structure of these molecules is illustrated in Figure 1. The temperature of the systems has been kept at $310 \mathrm{~K}$. The rectangular basic simulation box has consisted of 256 DPPC molecules, arranged in a bilayer containing 128 molecules in each of the two leaflets. To ensure full hydration, which requires the water to lipid molar ratio to exceed $29.1{ }^{62} 8132$ water molecules have been used. In the systems containing anesthetics 112 chloroform, 72 halothane, 192 diethyl ether, or 96 enflurane molecules have also been placed in the hydrocarbon region of the membrane. The number of the anesthetic molecules has been chosen in such a way that they correspond to roughly the same anesthetic mass density in all the four systems. The molar fraction of the anesthetic molecules used in the simulations well (i.e., by a factor of about 3-4) exceeds what is used under surgical conditions, in order to magnify any possible effect of them on the membrane properties, as suggested by Oh and 
Klein. ${ }^{18}$ For similar reasons, the pressure of 1000 bar used in the high pressure simulations is also considerably higher than what is needed for pressure reversal.

DPPC molecules have been modeled using a GROMOS87-based force field, ${ }^{63}$ developed specifically for lipids. According to this potential model, the $\mathrm{CH}, \mathrm{CH}_{2}$, and $\mathrm{CH}_{3}$ groups have been treated as united atoms. This model of DPPC is known to be in the biologically more relevant $\alpha$ phase at $T=310 \mathrm{~K}$ and $p=1$ bar. ${ }^{64}$ Chloroform, diethyl ether, and enflurane molecules have been described by the GROMOS96 force field, ${ }^{65-68}$ for halothane the potential model of Scharf and Laasonen ${ }^{69}$ has been used, whereas water molecules have been described by the rigid, three-site SPC potential. ${ }^{70}$ The geometry of the water molecules as well as all bond lengths of the DPPC and anesthetic molecules have been kept fixed by means of the SHAKE algorithm. ${ }^{71}$ Angular and torsional flexibility of DPPC and the anesthetics has been allowed, according to the force fields used. All interactions have been truncated to zero beyond the group-based center-center cut-off distance of $9.0 \AA$. The long range part of the electrostatic interaction has been accounted for using the smooth particle mesh Ewald (sPME) method. ${ }^{42}$

The simulations have been started from already equilibrated configurations obtained from our previous work, ${ }^{57}$ and have been performed using an in-house modified version of the GROMACS 5.1 molecular dynamics simulation package, ${ }^{72}$ which also distributes the lateral pressure among all the atoms present in the basic box. ${ }^{73}$ The equations of motion have been integrated in time steps of $2 \mathrm{fs}$, using the leapfrog algorithm. The temperature and pressure of the systems have been controlled by means of the Nosé-Hoover thermostat ${ }^{74,75}$ and ParrinelloRahman barostat, ${ }^{76}$ respectively, using the characteristic time of $0.5 \mathrm{ps}$ in both cases. Pressure has been controlled in an anisotropic way, namely separate couplings have been employed along all three spatial directions. The lateral pressure profile has been calculated over a $20 \mathrm{~ns}$ long equilibrium trajectory in each system, whereas for the calculation of the free volume 
characteristics 2000 sample configurations per system, separated by 10 ps long trajectories each, have been saved. Equilibrium snapshots of the ten systems simulated are shown in Figure 2. Finally, all profiles calculated have been averaged also over the two sides of the membrane, therefore, in the following they will always been shown only in one side of the membrane.

2.2. Calculation of the Lateral Pressure Profile. The calculation of the lateral pressure profile requires the decomposition of the pressure to contributions of well defined positions, preferably localized at the positions of the atoms. Any element of the global pressure tensor can be expressed as the sum of a kinetic, or ideal gas, and a potential, or excess contribution:

$$
p_{\mu v}=\frac{2}{V}\left(\frac{1}{2} \sum_{i} m_{i} v_{i}^{\mu} v_{i}^{v}-\Xi_{\mu v}\right),
$$

where $m_{\mathrm{i}}$ is the mass of the $i$ th particle, $v_{i}^{\mu}$ and $v_{i}^{v}$ are the $\mu$ and $v$ components of its velocity, $V$ is the total volume of the system, and $\Xi_{\mu \nu}$, corresponding to the excess contribution, is the respective element of the virial tensor. Clearly, the kinetic part of the pressure can trivially be decomposed to contributions of the individual atoms. However, in the case of pairwise additive forces the virial term can be written as an integral over an open path $C_{i j}$ connecting the pair of the interacting atoms $i$ and $j$, as

$$
\Xi_{\mu v}(\mathbf{r})=-\frac{1}{2}\left\langle\sum_{i j} f_{i j}^{\mu} \int_{C_{i j}} d s^{v} \delta(\mathbf{r}-\mathbf{s})\right\rangle
$$

Here we use the Harasima path, ${ }^{35}$ because it allows the distribution of the lateral pressure among the atomic positions (equally distributing the contributions coming from pair interactions between the positions of the two interacting atoms). ${ }^{37}$ Further, the lateral pressure 
can be localized this way even in the case of non-pairwise additive potential terms, such as the reciprocal space contribution of the Ewald summation or its particle mesh variants. ${ }^{36}$ Thus, in the particle mesh based variants of the Ewald method, one of which is used also here, the virial contribution is distributed on the mesh nodes as ${ }^{53}$

$$
\widetilde{\Xi}_{\mu \mu}^{\mathrm{rec}, i}\left(\mathbf{r}_{p}\right)=\mathrm{FFT}^{-1}\left\{B(\mathbf{m}) F F T[\tilde{\rho}] f\left(\mathbf{m}^{2}\right) g_{\mu \mu}(\mathbf{m})\right\},
$$

where FFT stands for fast Fourier transformation, ${ }^{77} \mathbf{r}_{p}$ is the position of a given mesh node, $f\left(\mathbf{m}^{2}\right)$ and $g_{\mu \mu}(\mathbf{m})$ are suitably chosen functions, ${ }^{53}$ the charge distribution can be given as

$$
\tilde{\rho}\left(\mathbf{r}_{p}\right)=\frac{1}{h} \sum_{i} W\left(\mathbf{r}_{p}-\mathbf{r}_{i}\right) q_{i},
$$

$W\left(\mathbf{r}_{p^{-}} \mathbf{r}_{i}\right)$ is a suitably chosen charge assignment function, $q_{i}$ is the charge of atom $i, h$ is the mesh spacing, and the function $B(\mathbf{m})$ depends on the specific interpolation scheme employed by the given variant of the particle mesh Ewald method. The virial contribution can then be interpolated back to obtain the contributions assigned to the individual atoms as

$$
\Xi_{\mu \mu}^{\mathrm{rec}, i}=\frac{q_{i}}{V} \sum_{r_{p}} \widetilde{\Xi}_{\mu \mu}^{\mathrm{rec}, i}\left(r_{p}\right) W\left(r_{i}-r_{p}\right)
$$

It should be noted that in these equations indices $\mu$ and $v$ can only stand for the $Y$ and $Z$ axes, being parallel with the plane of the membrane, not only because here we are interested in the profile of only the lateral contribution of the pressure, but also because the use of the Harasima path does not allow the calculation of the normal component of the local virial tensor.

Having the lateral pressure been decomposed to contributions coming from the individual atoms, $p_{\mathrm{L}}^{i}$, its profile along the membrane normal axis, $X$, can simply be calculated as 


$$
p_{\mathrm{L}}(X)=\frac{V}{A}\left\langle\sum_{i} p_{\mathrm{L}}^{i} \delta\left(X-X_{i}+X_{\mathrm{com}}\right)\right\rangle
$$

where $A$ is the cross-section area of the system, the brackets $<\ldots>$ denote ensemble averaging, whereas $X_{i}$ and $X_{\text {com }}$ are the positions of the $i$ th atom and of the center-of-mass of the lipid bilayer, respectively, along the $X$ axis.

2.3. Calculation of the Free Volume Characteristics. Free volume properties are traditionally analyzed using the Voronoi and Delaunay tessellations in the system of interest. ${ }^{58,59}$ However, in its original formulation the Voronoi-Delaunay method assumes that the system to be studied consists of points (or spheres of equal radii, such as in atomic systems). When studying molecular systems, which are built up by spherical particles (i.e., atomic sites) of different radii, the original method needs to be improved. To keep the underlying physical concept unchanged, one should use the Voronoi S-tessellation, ${ }^{78-80}$ where distance of a spatial point is measured from the surface rather than from the centre of the atoms. Hence, the Voronoi S-region is the locus of the spatial points that are closer to the surface of a given atom than to that of any other atoms. Besides the Voronoi S-region, the other important construction of the method is the Delaunay S-simplex (tetrahedron), defined by four mutually neighboring atoms. The radius of the largest empty sphere that can be inscribed between these atoms characterizes the size of the interstitial void located between them. The position and radius of these interstitial spheres can be calculated simultaneously with the tessellation itself, ${ }^{79}$ while the volume of the Delaunay simplexes themselves can easily be obtained by means of elementary geometry.

In contrast to the interstitial spherical cavities, the determination of the entire empty volume (of irregular shape) located between the atoms is a considerably more complicated task. First, while the ordinary Voronoi regions are convex polyhedra with flat faces, the 
Voronoi S-regions have curved faces, and thus their volume can only be calculated numerically. ${ }^{81}$ This makes the use of the Voronoi S-tessellation unsuitable for volumetric studies, since such studies need accurate and fast calculations. A reasonable compromise between computational cost and accuracy can be the use of the power (radical) VoronoiDelaunay tessellation, ${ }^{60,82,83}$ where the faces of the Voronoi regions are defined by the planes from the points of which the tangents drawn to the two spheres are of equal lengths. In system where the radii of the various atoms do not differ much from each other, such as in the present case, the power Voronoi tessellation retains the physical meaning of the original method, as in such cases the power Voronoi regions provide a very good approximation of the Voronoi Sregions. Another difficulty stems from the presence of strongly overlapping (i.e., chemically bound) atoms. Several approaches have been proposed previously to overcome this problem, involving either the calculation of the volume of the overlapped regions of the atoms, ${ }^{84}$ or that of the voids. ${ }^{85,86}$ In the present work, we use an approach based on Delaunay subsimplexes, ${ }^{87}$ in which the occupied volume of the Delaunay simplexes (and of the Voronoi regions with flat faces) are calculated with analytical formulas for arbitrary systems of overlapping atoms. ${ }^{86,88}$ Recently, this method was used to calculate the volumetric characteristics of proteins in aqueous solutions. ${ }^{89,90}$

An important characteristic of lipid bilayers is the profile of free volume along the axis perpendicular to the plane of the membrane. This profile can be calculated in several ways, e.g., by placing sample points in the system, either randomly or along a grid. ${ }^{91-94}$ Having the power Voronoi-Delaunay tessellation of the system determined, however, the profile of the free volume fraction, $\varepsilon$, can readily be calculated as the sum of the empty volumes of the Delaunay simplexes located in the different slices of the system, normalized by the total volume of these simplexes themselves (the position of a simplex being defined as that of its center). 


\section{RESULTS}

3.1. Lateral Pressure Profiles. The lateral pressure profiles obtained in the pure membrane at 1 bar as well as in the anesthetic containing membranes both at 1 and 1000 bar are shown and compared in Figure 3. The pressure profiles exhibit a constant plateau in the bulk aqueous phase around the $p_{\mathrm{L}}$ value corresponding to the overall pressure of the system. This plateau is followed by a clear maximum at about $X=20 \AA$, and a minimum around the $X$ value of $12-13 \AA$. The position of the peak corresponds to the dense region of the lipid headgroups, while that of the minimum is close to the average position of the $\mathrm{C}=\mathrm{O}$ groups of the lipid tails, ${ }^{57}$ i.e., the boundary of the apolar region of the membrane. In the hydrocarbon phase, the profile is again roughly constant, apart from the marked peak occurring at $X=0 \AA$, i.e., right in the middle of the membrane. It should be noted that here the density of the membrane is of minimum, ${ }^{57}$ therefore, the presence of this lateral pressure maximum cannot simply be explained by the effect of the density. Instead, it is sensible to assume that the outer part of the hydrocarbon phase is characterized by a rather homogeneous distribution of the atoms, which is reflected in the more or less constant value of $p_{\mathrm{L}}$. By contrast, in the middle of the membrane, i.e., in the region of the chain terminal $\mathrm{CH}_{3}$ groups, the spatial distribution of the atoms exhibits larger fluctuations, relatively large voids are surrounded by rather crowded patches, and the presence of these high density patches leads to the relatively high lateral pressure value. This explanation is also in accordance with our earlier finding that the middle of a phospholipid membrane is characterized by larger but considerably less spherical voids than the outer part of the hydrocarbon region. ${ }^{78}$ 
The increase of the pressure of the system leads, as expected, to the corresponding overall shift of the entire lateral pressure profile, without altering much of its shape. The inclusion of the anesthetic molecules has a much smaller effect on the lateral pressure profile. Nevertheless, it leads to a slight shift of the minimum position away from the membrane interior and, correspondingly, to a clear decrease of the lateral pressure in the $X$ range of 13-18 $\AA$ in every case. This range corresponds to the lipid side of the headgroup region, where the glycerol backbone of the DPPC molecules as well as the ester groups linking the fatty acid tails to this backbone are located. In this region, the behavior of the lateral pressure profile meets both of our criteria, namely, it is changed in the same way (i.e., decreased) upon the addition of all the anesthetic molecules considered, and it is changed in the opposite way (i.e., increased) by increasing the overall pressure. This change might thus be related to the molecular mechanism of anesthesia.

Since in calculating the lateral pressure profile the pressure contributions were distributed among the different atoms, ${ }^{37}$ it is straightforward to separate the contributions of the lipid, anesthetic, and water molecules to the entire $p_{\mathrm{L}}(X)$ profile. This separation is shown in Figure 4 for the anesthetic containing systems simulated at 1 bar. To further analyze the origin of the above discussed change of the $p_{\mathrm{L}}(X)$ profile in the $X$ range of 13-18 $\AA$, we have calculated the difference of the lipid, anesthetic, and water contributions to the $p_{\mathrm{L}}(X)$ profile between the anesthetic containing and pure membrane at 1 bar in every case. The obtained results are shown in Figure 5. The anesthetic molecules always increase the lateral pressure in the membrane interior, where they are located. In the middle of the membrane, the lipid contribution is decreased in the presence of anesthetics, presumably due to the lateral expansion of the membrane. ${ }^{57}$ In the $X$ range relevant for us, i.e., $13 \AA<X<18 \AA$, both the lipid and the water contribution to $p_{\mathrm{L}}(X)$ is decreased in the presence of anesthetics. Clearly, the anesthetic molecules themselves do not reach this part of the membrane, but they always 
stay somewhat closer to the middle of the bilayer. ${ }^{57}$ On the other hand, water molecules can penetrate into this region, ${ }^{95,96}$ as the $\mathrm{O}$ atoms and polar ester $(\mathrm{C}-\mathrm{O}-\mathrm{C}=\mathrm{O})$ groups of the lipid tails are located here. ${ }^{57}$ The observed decrease of the lateral pressure in this part of the membrane can be attributed to the fact that the anesthetic molecules are accumulated in the immediate vicinity of this region, but do not penetrate here, and hence the anesthetic-induced lateral expansion of the membrane ${ }^{57}$ is expected to be the strongest here.

3.2. Free Volume Properties. In analyzing the voids in the different membranes, the simplest characteristics to be discussed is the size distribution of the elemental spherical vacancies located between the atoms. As it was described above, these interstitial voids are determined by the Delaunay tessellation of the system, namely each void corresponds to the center of the Delaunay simplex formed by four mutually neighboring atoms. ${ }^{78}$ This fact allows us not only to determine these spherical voids, but also to assign them to different parts of the system according to the atoms forming the corresponding Delaunay simplexes. Thus, here we defined an elemental void to belong to the lipid phase of the system if all the four atoms forming its Delaunay simplex belong to a DPPC (or anesthetic) molecule. Similarly, voids corresponding to Delaunay simplexes formed by four water atoms are regarded to belong to the water phase of the system, whereas those corresponding to Delaunay simplexes formed both by lipid and water atoms are regarded to belong to the interfacial region of the system.

The distribution of the radius $R_{\mathrm{i}}$ of the elemental interstitial voids belonging to these three different parts of the system is shown in Figure 6 as obtained in the pure membrane at 1 bar as well as in the membranes containing chloroform both at 1 and 1000 bar. (Similar distributions have been obtained with the other anesthetic molecules, as well.) As is seen, the inclusion of anesthetics in the membrane does not alter the $P\left(R_{\mathrm{i}}\right)$ distributions in the water 
phase and in the interfacial region, but shifts the distribution to slightly higher values in the lipid part of the system. This finding is not surprising, since the anesthetic molecules are located deeply inside the hydrocarbon phase of the membrane. Further, as it is expected, the increase of the pressure shifts all these distributions to lower values.

Table 1 shows the empty volume corresponding to the lipid, anesthetic, and water molecules, as obtained in the different systems simulated. As it was described above, the total volume of the Voronoi cell of an atom can be divided into "occupied" and "empty" parts, the former being covered by the given atom itself, while the latter not. Since the occupied volume of a Voronoi region simply reflects the size of its central atom, it is sensitive neither to the pressure nor to the presence of the anesthetics. The amount of the empty volume, on the other hand, is characteristic of the local arrangement of the atoms. As it is seen from Table 1, the empty volume corresponding both to the lipid and to the water molecules agree, within error bars, as obtained in the pure and anesthetic containing membranes at 1 bar in every case. On the other hand, considerably large empty volume is associated with the anesthetic molecules themselves in every case. In other words, the increase of the empty volume corresponding to the inclusion of the anesthetic molecules, evidenced by the lateral expansion of the membrane,${ }^{57}$ is localized to the immediate vicinity of the anesthetic molecules. This finding is in line with our previous result that the presence of anesthetic molecules induces a decrease of the lateral pressure in the membrane region located in the immediate vicinity of the preferred position of the anesthetic molecules. Finally, as it is expected, the increase of the pressure leads to a marked decrease of the empty volume in every case, reverting thus the increase of the empty volume associated with the presence of the anesthetic molecules.

The profile of the free volume fraction, $\varepsilon$, across the membrane is shown in Figure 7, again as obtained in the pure membrane at 1 bar as well as in the anesthetic containing membranes both at 1 and 1000 bar. Clearly, the increase of the pressure leads to the decrease 
of the empty volume fraction across the entire membrane. The inclusion of the anesthetic molecules in the membrane has a more subtle effect on the free volume profile. In general, it decreases the fraction of free volume, or leaves it unchanged at the aqueous side, and increases it at the lipid side of the dense headgroup region. Although there are several differences between the behavior of the different anesthetic molecules in this respect, the fraction of the empty volume is noticeably increased in their presence in the $X$ range between about $8 \AA$ and $16 \AA$ in every case. This $X$ range largely overlaps with that corresponding to the decrease of the lateral pressure (i.e., between about $13 \AA$ and $18 \AA$ ), but extends also somewhat towards the membrane region in which the anesthetic molecules are located. It should be noted that, as described in our previous paper, ${ }^{57}$ anesthetic molecules prefer two distinct positions along the membrane normal axis in every case, namely the middle of the membrane, and the $X$ range around $10 \AA$, although the relative weights of these two preferences can vary from anesthetic to anesthetic. ${ }^{29,57,97}$ Consistently, the fraction of the free volume is typically increased by the anesthetic molecules in both of these two regions; however, it is only the outer of these two regions in which all the anesthetic molecules considered leads to a clear increase of it.

Thus, our findings indicate that the increase of the empty volume in the membrane in the presence of anesthetics, associated with the anesthetic molecules themselves, leads to an overall increase of the fraction of empty volume in the membrane region where the anesthetic molecules are located, and also in the immediate vicinity of this region at its headgroup side. This results in a decrease of the lateral pressure in the region of the ester groups of the DPPC molecules, located right next to the membrane region in which the anesthetic molecules are accumulated. These changes are clearly reverted by the increase of the pressure, and hence they might be behind the molecular mechanism of anesthesia. 


\section{DISCUSSION AND SUMMARY}

In our previous study we found that anesthetic induced lateral expansion of the membrane might be a process that is relevant for the molecular mechanism of anesthesia, because such a lateral expansion was observed upon the introduction of all anesthetic molecules considered, and this effect was clearly reverted by the increase of the pressure. ${ }^{57}$ This finding was also consistent with the more than sixty years old critical volume hypothesis of Mullins. ${ }^{6}$ In the present study we analyzed in detail the origin of this anesthetic induced lateral expansion, and related it also to the hypothesis of Cantor, who claimed that anesthetics induce some changes in the lateral pressure profile of the lipid membrane, which alters the conformational equilibrium of certain membrane-bound proteins. ${ }^{13}$ In order to calculate the lateral pressure profile we used a computationally feasible method recently developed by us, which takes accurately into account also the lateral pressure contribution coming from the (non-pairwise additive) long range correction part of the smooth Particle Mesh Ewald method, and distributes the lateral pressure contributions among the different atomic sites. ${ }^{53}$

Previously, we also showed that anesthetics prefer two distinct positions in the membrane. The first of these positions is right in the middle of the membrane, while the second one is located at the outer edge of the hydrocarbon phase, close to the region of the $\mathrm{C}=\mathrm{O}$ groups of the lipid tails. ${ }^{57}$ This finding was in agreement with the results of earlier coarse-grain simulations, ${ }^{97}$ and was later confirmed also by Chen et al. ${ }^{29}$ In the present study we have demonstrated that anesthetics located close to the $\mathrm{C}=\mathrm{O}$ groups might be related to the aforementioned lateral expansion, and thus be responsible for the molecular mechanism of anesthesia. Thus, although in the presence of certain anesthetics the fraction of the empty volume is increased around both of these preferred positions, it is only at the outer position 
that this increase of empty volume occurs in the presence of all anesthetics considered. Further, the presence of anesthetics is found not to alter the lateral pressure in the middle of the membrane, but to decrease it in the region of the ester groups of the lipid tails, very close to the outer preferred position of the anesthetic molecules.

We have shown here that the additional free volume occurring in the membrane upon addition of the anesthetics is localized right around the anesthetic molecules themselves, and thus, the empty volume increases in the region where anesthetics are preferentially located. This increase of the empty volume results in a decrease of the lateral pressure in the nearby region of the ester groups, where the anesthetic molecules do not penetrate. In other words, the lateral pressure decreases where the lateral expansion induced by the presence of anesthetics is not accompanied by the occurrence of additional (i.e., anesthetic) molecules. This way, we have found a link between the critical volume hypothesis of Mullins ${ }^{6}$ and the lateral pressure hypothesis of Cantor. ${ }^{13}$ Thus, by contrast to earlier claims, ${ }^{11,12,14}$ the increase of the membrane volume upon addition of anesthetics is caused by the lateral expansion rather than the thickening of the membrane. ${ }^{57}$ This lateral expansion is the strongest in the membrane region of the ester groups, where anesthetics do not penetrate, but it is right next to the outer of their two preferred positions. This leads to a clear decrease of the lateral pressure in this region, an effect that can simply be reverted by the increase of the overall pressure. As a consequence, given that indeed this change of the lateral pressure profile lays behind the molecular mechanism of anesthesia, the conformational changes of the relevant membrane bound protein molecules, predicted by Cantor, ${ }^{13}$ are also expected to occur in this region of the ester groups of the membrane.

\section{ACKNOWLEDGEMENTS}


This work has been supported by the Hungarian NKFIH Foundation under Project No. 119732, by RFBR grant No. 15-03-03329, and by the Action Austria-Hungary Foundation under project No. 93öu3. The calculations have been performed using the Vienna Scientific Cluster (VSC).

\section{REFERENCES}

(1) Kao, C. Y. Tetrodotoxin, Saxitoxin and Their Significance in the Study of Excitation Phenomena. Pharmacol. Rev. 1966, 18, 987-1049.

(2) Lee, A. G. Model for Action of Local Anaesthetics. Nature 1976, 262, 545-548.

(3) Franks, N. P.; Lieb, W. R. Where Do General Anaesthetics Act? Nature 1978, 274, 339342.

(4) Franks, N. P.; Lieb, W. R. Molecular and Cellular Mechanisms of General Anaesthesia. Nature 1994, 367, 607-614.

(5) Mitchell, D. C.; Lawrence, J. T. R.; Litman, B. J. Primary Alcohols Modulate the Activation of the G Protein-Coupled Receptor Rhodopsin by a Lipid-Mediated Mechanism. J. Biol. Chem. 1996, 271, 19033-19036.

(6) Mullins, L. J. Some Physical Mechanisms in Narcosis. Chem. Rev. 1954, 54, 289-323.

(7) Johnson, S. M.; Miller, K. W. Antagonism of Pressure and Anaesthesia. Nature 1970, 228, 75-76.

(8) Jain, M. K.; Wu, N. Y.; Wray, L. V. Drug-Induced Phase Change in Bilayer as Possible Mode of Action of Membrane Expanding Drug. Nature 1975, 255, 494-496.

(9) Miller, K. W.; Pang, K. General Anaesthetics Can Selectively Perturb Lipid Bilayer Membranes. Nature 1976, 263, 253-255. 
(10) Boggs, J. M.; Yoong, T.; Hsia, J. C. Site and Mechanism of Anesthetic Action. Mol. Pharmacol. 1976, 12, 127-135.

(11) Haydon, D. A.; Hendry, B. M.; Levinson, S. R.; Requena, J. The Molecular Mechanisms of Anaesthesia. Nature 1977, 268, 356-358.

(12) Ashcroft, R. G.; Coster, H. G. L.; Smith, J. R. Local Anaesthetic Benzyl Alcohol Increases Membrane Thickness. Nature 1977, 269, 819-820.

(13) Cantor R. S. The Lateral Pressure Profile in Membranes: A Physical Mechanism of General Anesthesia. Biochemistry 1997, 36, 2339-2344.

(14) Ashcroft, R. G.; Coster, H. G. L.; Smith, J. R. The Molecular Organisation of Bimolecular Lipid Membranes. The Effect of Benzyl Alcohol on the Structure. Biochim. Biophys. Acta 1977, 469, 13-22.

(15) Trudell, J. R.; Payan, D. G.; Chin, J. H., Cohen, E. N. The Antagonistic Effect of an Inhalation Anesthetic and High Pressure on the Phase Diagram of Mixed DipalmitoylDimyristoylphosphatidylcholine Bilayers Proc. Natl. Acad. Sci. USA 1975, 72, 210213.

(16) Forrest, B. J.; Rodham, D. K. An Anaesthetic-Induced Phosphatidylcholine Hexagonal Phase. Biochim. Biophys. Acta 1985, 814, 281-288.

(17) Huang, P.; Bertaccini, E.; Loew, G. H. Molecular Dynamics Simulation of AnestheticPhospholipid Bilayer Interactions. J. Biomol. Struct. Dyn. 1995, 12, 725-754.

(18) Oh, K. J.; Klein, M. L. Effects of Halothane on Dimyristoylphosphatidylcholine Lipid Bilayer Structure: A Molecular Dynamics Simulation Study. Bull. Korean Chem. Soc. 2009, 30, 2087-2092.

(19) Reigada, R. Influence of Chloroform in Liquid-Ordered and Liquid-Disordered Phases in Lipid Membranes. J. Phys. Chem. B 2011, 115, 2527-2535. 
(20) Moskovitz, Y.; Yang, H. Modelling of Noble Anaesthetic Gases and High Hydrostatic Pressure Effects in Lipid Bilayers. Soft Matter 2015, 11, 2125-2138.

(21) Turner, G. L.; Oldfield, E. Effect of a Local Anaesthetic on Hydrocarbon Chain Order in Membranes. Nature 1979, 277, 669-670.

(22) Franks, N. P.; Lieb, W. R. The Structure of Lipid Bilayers and the Effects of General Anaesthetics. J. Mol. Biol. 1979, 133, 469-500.

(23) López Cascales, J. J.; Hernández Cifre, J. G.; García de la Torre, J. Anaesthetic Mechanism on a Model Biological Membrane: A Molecular Dynamics Simulation Study. J. Phys. Chem. B 1998, 102, 625-631.

(24) Tu, K.; Tarek, M.; Klein, M. L.; Scharf, D. Effects of Anaesthetics on the Structure of a Phospholipid Bilayer: Molecular Dynamics Investigation of Halothane in the Hydrated Liquid Crystal Phase of Dipalmitoylphosphatidylcholine. Biophys. J. 1998, $75,2123-2134$

(25) Chau, P. L.; Hoang, P. N. M.; Picaud, S.; Jedlovszky, P. Pressure Reversal of General Anaesthetics: A Possible Mechanism from Molecular Dynamics Simulations. J. Mol. Liquids 2009, 147, 128-134.

(26) Trudell, J. R.; Hubbell, W. L.; Cohen, E. N. The Effect of Two Inhalation Anesthetics on the Order of Spin-Labelled Phospholipid Vesicles. Biochim. Biophys. Acta 1973, $291,321-327$

(27) Koubi, L.; Tarek, M.; Klein, M. L.; Scharf, D. Distribution of Halothane in a Dipalmitoylphosphatidylcholine Bilayer from Molecular Dynamics Calculations. Biophys. J. 2000, 78, 800-811.

(28) Porasso, R. D.; Drew Bennett, W. F.; Oliveira-Costa, S. D.; López-Cascales, J. J. Study of the Benzocaine Transfer from Aqueous Solution to the Interior of a Biological Membrane. J. Phys. Chem. B 2009, 113, 9988-9994. 
(29) Chen, J.; Chen, L.; Wang, Y.; Wang, X.; Zeng, S. Exploring the Effects on Lipid Bilayer Induced by Noble Gases via Molecular Dynamics Simulations. Sci. Rep. 2015, $5,17235-1-6$.

(30) Johnson, F. H.; Flagler, E. A. Hydrostatic Pressure Reversal of Narcosis in Tadpoles. Science 1950, 112, 91-92.

(31) Lever, M. J.; Miller, K. W.; Paton, W. D. M.; Smith, E. B. Pressure Reversal of Anaesthesia. Nature 1971, 231, 368-371.

(32) Halsey, M. J.; Wardley-Smith, B. Pressure Reversal of Narcosis Produced by Anaesthetics, Narcotics and Tranquillisers. Nature 1975, 257, 811-813.

(33) Schofield, P.; Henderson, J. R. Statistical Mechanics of Inhomogeneous Fluids. Proc. R. Soc. Lond. A 1982, 379, 231-246.

(34) Irving, J. H.; Kirkwood, J. G. The Statistical Mechanical Theory of Transport Processes. IV. The Equations of Hydrodynamics. J. Chem. Phys. 1950, 18, 817-829.

(35) Harasima, A. Molecular Theory of Surface Tension. Adv. Chem. Phys. 1958, 1, 203237.

(36) Sonne, J.; Hansen, F. Y.; Peters, G. H. Methodological Problems in Pressure Profile Calculations for Lipid Bilayers. J. Chem. Phys. 2005, 122, 124903-1-9.

(37) Sega, M.; Fábián, B.; Jedlovszky, P. Layer-by-Layer and Intrinsic Analysis of Molecular and Thermodynamic Properties Across Soft Interfaces. J. Chem. Phys. 2015, 143, 114709-1-8

(38) Ewald, P. Die Berechnung Optischer und Elektrostatischer Gitterpotentiale. Ann. Phys. 1921, 369, 253-287.

(39) de Leeuw, S. W.; Perram, J. W.; Smith, E. R. Simulation of Electrostatic Systems in Periodic Boundary Conditions. I. Lattice Sums and Dielectric Constants. Proc. R. Soc. Lond. A 1980, 373, 27-56. 
(40) Allen, M. P.; Tildesley, D. J. Computer Simulation of Liquids; Clarendon Press: Oxford, 1987.

(41) Darden, T.; York, D.; Pedersen, L. Particle Mesh Ewald: An N·log(N) Method for Ewald Sums in Large Systems. J. Chem. Phys. 1993, 98, 10089-10092.

(42) Essman, U.; Perera, L.; Berkowitz, M. L.; Darden, T.; Lee, H.; Pedersen, L. G. A Smooth Particle Mesh Ewald Method. J. Chem. Phys. 1995, 103, 8577-8594.

(43) Hockney, R. W.; Eastwood, J. W. Computer Simulation Using Particles; Taylor and Francis: New York, 1988.

(44) Lindahl, E.; Edholm, O. Spatial and Energetic-Entropic Decomposition of Surface Tension in Lipid Bilayers from Molecular Dynamics Simulations. J. Chem. Phys. 2000, 113, 3882-3893.

(45) Gullingsrud, J.; Schulten, K. Lipid Bilayer Pressure Profiles and Mechanosensitive Channel Gating. Biophys. J. 2004, 86, 3496-3509.

(46) Patra, M. Lateral Pressure Profiles in Cholesterol-DPPC Bilayers. Eur. Biophys. J. 2005, 35, 79-88.

(47) Carrillo-Trip, M.; Fellner, S. E. Evidence for a Mechanism by Which $\omega-3$ Polyunsaturated Lipids May Affect Membrane Protein Function. Biochemistry 2005, 44, 10164-10169.

(48) Ollila, O. H. S.; Róg, T.; Karttunen, M.; Vattulainen, I. Role of Sterol Type on Lateral Pressure Profiles of Lipid Membranes Affecting Membrane Protein Functionality: Comparison between Cholesterol, Desmosterol, 7-Dehydrocholesterol and Ketosterol. J. Struct. Biol. 2007, 159, 311-323.

(49) Ollila, S.; Hyvönen, M. T.; Vattulainen, I. Polyunsaturation in Lipid Membranes: Dynamic Properties and Lateral Pressure Profiles. J. Phys. Chem. B 2007, 111, 31393150. 
(50) Terama, E.; Ollila, O. H. S.; Salonen, E.; Rowat, A. C.; Trandum, C.; Westh, P.; Patra, M.; Karttunen, M.; Vattulainen, I. Influence of Ethanol on Lipid Membranes: from Lateral Pressure Profiles to Dynamics and Partitioning. J. Phys. Chem. B 2008, 112, 4131-4139.

(51) Griepernau, B.; Böckmann, R. A. The Influence of 1-Alkanols and External Pressure on the Lateral Pressure Profiles of Lipid Bilayers. Biophys. J. 2008, 95, 5766-5778.

(52) Booker, R. D.; Sum, A. K. Biophysical Changes Induced by Xenon on Phospholipid Bilayers. Biochim. Biophys. Acta 2013, 1828, 1347-1356.

(53) Sega, M. Fábián, B.; Jedlovszky, P. Pressure Profile Calculation with Mesh Ewald Methods. J. Chem. Theory Comput. 2016, 12, 4509-4515.

(54) Vanegas, J. M.; Torres-Sánchez, A.; Arroyo, M. Importance of Force Decomposition for Local Stress Calculations in Biomembrane Molecular Simulations. J. Chem. Theory Comput. 2014, 10, 691-702.

(55) Sega, M. Fábián, B.; Horvai, G.; Jedlovszky, P. How Is the Surface Tension of Various Liquids Distributed along the Interface Normal? J. Phys. Chem. B 2016, 120, $27468-27477$.

(56) Darvas, M.; Hoang, P. N. M.; Picaud, S.; Sega, M.; Jedlovszky, P. Anesthetic Molecules Embedded in a Lipid Membrane: A Computer Simulation Study. Phys. Chem. Chem. Phys. 2012, 14, 12956-12969.

(57) Fábián, B.; Darvas, M.; Picaud, S.; Sega, M.; Jedlovszky, P. The Effect of Anesthetics on the Properties of a Lipid Membrane in the Biologically Relevant Phase: A Computer Simulation Study. Phys. Chem. Chem. Phys. 2015, 17, 14750-14760.

(58) Voronoi, G. F. Recherches sur le Paralléloèders Primitives. J. Reine Angew. Math. 1908, 134, 198-287. 
(59) Delaunay, B. N. On the Emptiness of the Sphere, Izv. Akad. Nauk. SSSR, Otd. Math. Est. Nauk. 1934, 7, 793-800.

(60) Okabe, A.; Boots, B.; Sugihara, K.; Chiu, S. N. Spatial Tessellations: Concepts and Applications of Voronoi Diagrams, John Wiley: Chichester, 2000.

(61) Medvedev, N. N. The Voronoi-Delaunay Method in the Structural Investigation of Non-Crystalline Systems, SB RAS: Novosibirsk, 2000, in Russian.

(62) Nagle, J. F.; Zhang, R.; Tristram-Nagle, S.; Sun, W.; Petrache, H. I.; Suter, R. M. XRay Structure Determination of Fully Hydrated $L_{\alpha}$ Phase Dipalmitoylphosphatidylcholine Bilayers. Biophys. J. 1996, 70, 1419-1431.

(63) O Berger, O.; Edholm, O.; Jähnig, F. Molecular Dynamics Simulations of a Fluid Bilayer of Dipalmitoylphosphatidylcholine at Full Hydration, Constant Pressure, and Constant Temperature. Biophys. J. 1997, 72, 2002-2013.

(64) Leekumjorn, S.; Sum, A. K. Molecular Studies of the Gel to Liquid-Crystalline Phase Transition for Fully Hydrated DPPC and DPPE Bilayers. Biochim. Biophys. Acta 2007, 1768, 354-365.

(65) van Gunsteren, W. F.; Billeter, S. R.; Eising, A. A.; Hünenberger, P. H.; Krüger, P.; Mark, A. E.; Scott, W. R. P.; Tironi, I. G. Biomolecular Simulation: The GROMOS96 Manual and User Guide; vdf Hochschulverlag A.G. an der ETH Zürich and BIOMOS b.v.: Zürich, Groningen, 1996.

(66) Schuler, L. D.; Daura, X.; van Gunsteren, W. F. An Improved GROMOS96 Force Field for Aliphatic Hydrocarbons in the Condensed Phase. J. Comp. Chem. 2001, 22, $1205-1218$.

(67) Schuettelkopf, A. W.; van Aalten, D. M. F. PRODRG: a Tool for High-Throughput Crystallography of Protein-Ligand Complexes. Acta Cryst. D 2004, 60, 1355-1363. 
(68) Oostenbrink, C.; Villa, A.; Mark, A. E.; van Gunsteren, W. F. A Biomolecular Force Field Based on the Free Enthalpy of Hydration and Solvation: The GROMOS ForceField Parameter Sets 53A5 and 53A6. J. Comp. Chem. 2004, 25, 1656-1676.

(69) Scharf, D.; Laasonen, K. Structure, Effective Pair Potential and Properties of Halothane. Chem, Phys. Letters 1996, 258, 276-282.

(70) Berendsen, H. J. C.; Postma, J. P. M.; van Gunsteren, W. F.; Hermans, J. Interaction Models for Water in Relation to Protein Hydration. In Intermolecular Forces; Pullman, B., Ed.; Reidel: Dordrecht, 1981, p. 331-342.

(71) Ryckaert, J. P.; Ciccotti, G.; Berendsen, H. J. C. Numerical Integration of the Cartesian Equations of Motion of a System with Constraints: Molecular Dynamics Simulation of n-Alkanes. J. Comp. Phys. 1977, 23, 327-341.

(72) Pronk, S.; Páll, S.; Schulz, R.; Larsson, P.; Bjelkmar, P.; Apostolov, R.; Shirts, M. R.; Smith, J. C.; Kasson, P. M.; van der Spoel; D., et al. GROMACS 4.5: A HighThroughput and Highly Parallel Open Source Molecular Simulation Toolkit. Bioinformatics 2013, 29, 845-854.

(73) The code is freely available at https://github.com/Marcello-Sega/gromacs/tree/virial/.

(74) Nosé, S. A Molecular Dynamics Method for Simulations in the Canonical Ensemble. Mol. Phys. 1984, 52, 255-268.

(75) Hoover, W. G. Canonical Dynamics: Equilibrium Phase-Space Distributions. Phys. Rev. A 1985, 31, 1695-1697.

(76) Parrinello, M.; Rahman, A. Polymorphic Transitions in Single Crystals: A New Molecular Dynamics Method. J. Appl. Phys. 1981, 52, 7182-7190.

(77) Cooley, J. W.; Tukey, J. W. An Algorithm for the Machine Calculation of Complex Fourier Series. Math. Comput. 1965, 19, 297-301. 
(78) Alinchenko, M. A.; Anikeenko, A. V.; Medvedev, N. N.; Voloshin, V. P.; Mezei, M.; Jedlovszky, P. Morphology of Voids in Molecular Systems. A Voronoi-Delaunay Analysis of a Simulated DMPC Membrane. J. Phys. Chem. B. 2004, 108, 1905619067.

(79) Medvedev N. N.; Voloshin V. P.; Luchnikov, V. A.; Gavrilova M. L. An Algorithm for Three-Dimensional Voronoi S-Network. J. Comp. Chem. 2006, 27, 1676-1692.

(80) Voloshin, V. P.; Medvedev, N. N.; Andrews, M. N.; Burri, R. R.; Winter, R.; Geiger, A. Volumetric Properties of Hydrated Peptides: Voronoi-Delaunay Analysis of Molecular Simulation Runs. J. Phys. Chem. B 2011, 115, 14217-14228.

(81) Medvedev, N. N.; Voloshin, V. P. Open Problem: A Formula for Calculation of the Voronoi S-Region Volume. In Proceedings of the 10th International Symposium on Voronoi Diagrams in Science and Engineering (ISVD 2013); IEEE: Washington, D.C., 2013, pp. 89-91.

(82) Mezei, M. Modified Proximity Criteria for the Analysis of the Solvation of a Polyfunctional Solute. Mol. Simul. 1988, 1, 327-332.

(83) Aurenhammer, F.; Klein, R.; Lee, D. T. Voronoi Diagrams and Delaunay Triangulations, World Scientific: Singapore, 2013.

(84) Cazals, F.; Kanhere, H.; Loriot, S. Computing the Volume of a Union of Balls: A Certified Algorithm. ACM Trans. Math. Software 2011, 38, 3-1-20.

(85) Liang, J.; Edelsbrunner, H.; Fu, P.; Sudhakar, V.; Subramaniam, S. Analytical Shape Computation of Macromolecules, I: Molecular Area and Volume Through Alpha Shape. Proteins: Struct. Func. Genet. 1998, 33, 1-17.

(86) Voloshin, V. P., Anikeenko, A. V., Medvedev, N. N., Geiger, A. An Algorithm for the Calculation of Volume and Surface of Unions of Spheres. Application for Solvation 
Shells. In Proceedings of the 8th International Symposium on Voronoi Diagrams in Science and Engineering (ISVD 2011); IEEE: Washington, D.C., 2011, pp. 170-176.

(87) Sastry, S.; Corti, D. S.; Debenedetti, P.; Stillinger, F. H. Statistical Geometry of Particle Packings. I. Algorithm for Exact Determination f Connectivity, Volume, and Surface Areas of Void Space in Monodisperse and Polydisperse Sphere Packings. Phys. Rev. E 1997, 56, 5524-5532.

(88) Voloshin, V. P., Medvedev, N. N., Geiger, A. Fast Calculation of the Empty Volume in Molecular Systems by the Use of Voronoi-Delaunay Subsimplexes. In Transactions on Computational Science XXII; Gavrilova, M. L.; Kenneth Tan, C. J., Eds.; Springer: Berlin, 2014, pp. 156-172.

(89) Voloshin, V. P.; Kim, A. V.; Medvedev, N. N.; Winter, R.; Geiger, A. Calculation of the Volumetric Characteristics of Biomacromolecules in Solution by the VoronoiDelaunay Technique. Biophys. Chem. 2014, 192, 1-9.

(90) Voloshin, V. P.; Medvedev N. N.; Smolin, N. Geiger A.; Winter R. Exploring Volume, Compressibility and Hydration Changes of Folded Proteins Upon Compression. Phys. Chem. Chem. Phys. 2015, 17, 8499-8508.

(91) Marrink, S. J.; Sok, R. M.; Berendsen, H. J. C. Free Volume Properties of a Simulated Lipid Membrane. J. Chem. Phys. 1996, 104, 9090-9099.

(92) Jedlovszky, P.; Mezei, M. Calculation of the Free Energy Profile of $\mathrm{H}_{2} \mathrm{O}, \mathrm{O}_{2}, \mathrm{CO}$, $\mathrm{CO}_{2}, \mathrm{NO}$, and $\mathrm{CHCl}_{3}$ in a Lipid Bilayer with a Cavity Insertion Variant of the Widom Method. J. Am. Chem. Soc. 2000, 122, 5125-5131.

(93) Falck, E.; Patra, M.; Karttunen, M.; Hyvönen, M. T., Vattulainen, I. Impact of Cholesterol on Voids in Phospholipid Membranes. J. Chem. Phys. 2004, 121, 1267612689. 
(94) Kupiainen, M.; Falck, E.; Ollila, S.; Niemelä, P.; Gurtovenko, A. A.; Hyvönen, M. T., Patra, M.; Karttunen, M.; Vattulainen, I. Free Volume Properties of Sphingomyelin, DMPC, DPPC, and PLPC Bilayers. J. Comp. Theor. Nanosc. 2005, 2, 401-413.

(95) Jedlovszky, P.; Mezei, M. Grand Canonical Ensemble Monte Carlo Simulation of a Lipid Bilayer Using Extension Biased Rotations. J. Chem. Phys. 1999, 111, 1077010773.

(96) Jedlovszky, P.; Mezei, M. Orientational Order of the Water Molecules Across a Fully Hydrated DMPC Bilayer: A Monte Carlo Simulation Study. J. Phys. Chem. B. 2001, 105, 3614-3623.

(97) Pickholz, M.; Saiz, L.; Klein, M. L. Concentration Effects of Volatile Anesthetics on the Properties of Model Membranes: A Coarse-Grain Approach. Biophys. J. 2005, 88, $1524-1534$. 


\section{Tables}

TABLE 1. Empty Volume (in Units of $10^{6} \AA^{3}$ ) Associated with the Different Molecules in the Different Membranes Simulated

\begin{tabular}{ccccccccc}
\hline \hline & \multicolumn{2}{c}{ DPPC } & & \multicolumn{2}{c}{ anesthetic } & & \multicolumn{2}{c}{ water } \\
\cline { 2 - 3 } & 1 bar & 1000 bar & & 1 bar & 1000 bar & & 1 bar & 1000 bar \\
\hline pure membrane & $105.1 \pm 0.9$ & $88.1 \pm 0.8$ & & 0 & 0 & & $110.8 \pm 0.6$ & $101.4 \pm 0.6$ \\
membrane with CF & $108.0 \pm 0.9$ & $90.0 \pm 0.7$ & & $9.2 \pm 0.2$ & $7.5 \pm 0.1$ & & $110.9 \pm 0.7$ & $101.4 \pm 0.6$ \\
membrane with HAL & $105.7 \pm 0.9$ & $84.8 \pm 0.8$ & & $5.6 \pm 0.1$ & $4.6 \pm 0.1$ & & $110.8 \pm 0.7$ & $101.2 \pm 0.6$ \\
membrane with DE & $104.9 \pm 0.8$ & $91.5 \pm 0.6$ & & $13.2 \pm 0.2$ & $11.3 \pm 0.1$ & & $110.6 \pm 0.6$ & $101.3 \pm 0.6$ \\
membrane with ENF & $104.5 \pm 0.9$ & $88.3 \pm 0.6$ & & $7.8 \pm 0.2$ & $6.5 \pm 0.1$ & & $110.7 \pm 0.7$ & $101.3 \pm 0.6$ \\
\hline \hline
\end{tabular}




\section{Figure legends}

Figure 1. Schematic structure of DPPC and of the anesthetic molecules considered.

Figure 2. Equilibrium snapshot of the ten systems simulated. The C, O, N, and P atoms of the DPPC molecules are shown by green, red, light blue, and yellow colors, respectively. Water molecules are shown by red thin sticks, while anesthetic molecules are shown enlarged for better visibility (CF: green, HAL: dark blue, DE: red, ENF: olive).

Figure 3. Comparison of the lateral pressure profile across the pure lipid membrane at 1 bar (solid curves) with the membranes containing anesthetics, simulated at 1 bar (filled circles) and 1000 bar (open circles). Top panel, orange color: membrane containing $\mathrm{CF}$, second panel, red color: membrane containing HAL, third panel, green color: membrane containing DE, bottom panel, blue color: membrane containing ENF. $X=0 \AA$ corresponds to the middle of the bilayer. Error bars are always smaller than the symbols.

Figure 4. Total lateral pressure profile across the membranes containing CF (top panel), HAL (second panel), DE (third panel), and ENF (bottom panel), simulated at 1 bar (black open circles), along with the contributions given by the DPPC (brown solid lines), anesthetic (violet solid lines) and water molecules (cyan solid lines). $X=0 \AA$ corresponds to the middle of the bilayer.

Figure 5. Difference between the lateral pressure profile contributions of the DPPC (full circles), anesthetic (solid curves), and water molecules (open circles) as obtained in the anesthetic containing and pure membranes at 1 bar. Top panel, orange color: membrane containing CF, second panel, red 
color: membrane containing HAL, third panel, green color: membrane containing DE, bottom panel, blue color: membrane containing ENF. $X=0 \AA$ corresponds to the middle of the bilayer.

Figure 6. Distribution of the radius of the elemental spherical interstitial voids in the lipid phase (brown), interfacial region (magenta), and water phase (cyan) of the pure membrane simulated at 1 bar (solid curves), as well as in the chloroform containing membranes simulated at 1 bar (full circles) and 1000 bar (open circles).

Figure 7. Profile of the fraction of empty volume in pure lipid membrane at 1 bar (solid curves), compared with that in the membranes containing anesthetics, simulated at 1 bar (filled circles) and 1000 bar (open circles). Top panel, orange color: membrane containing CF, second panel, red color: membrane containing HAL, third panel, green color: membrane containing DE, bottom panel, blue color: membrane containing ENF. $X=0 \AA$ corresponds to the middle of the bilayer. Error bars are always smaller than the symbols. 

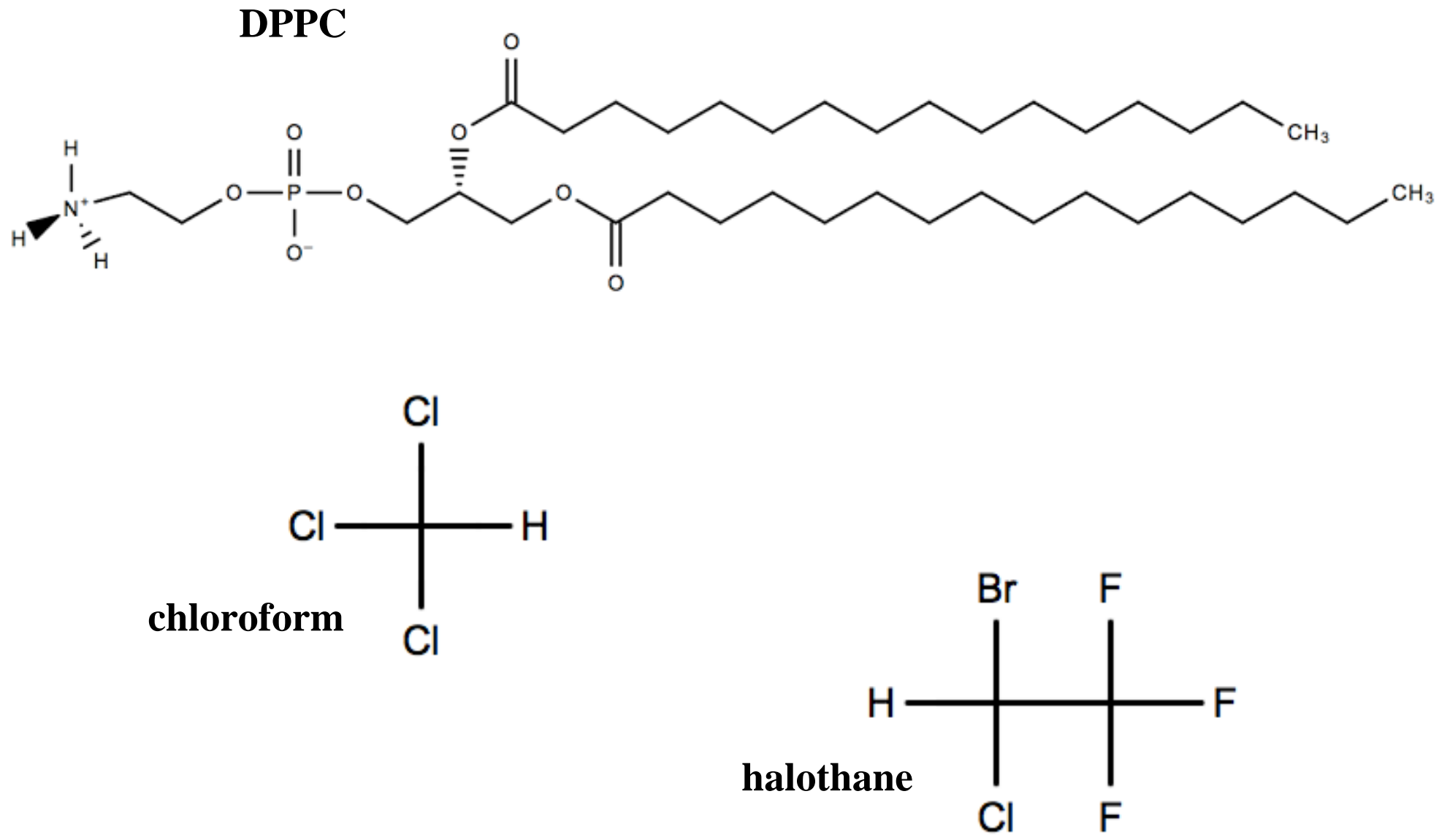

$\mathrm{H}_{3} \mathrm{C}-\mathrm{CH}_{2}-\mathrm{O}-\mathrm{CH}_{2}-\mathrm{CH}_{3}$ diethyl ether

enflurane

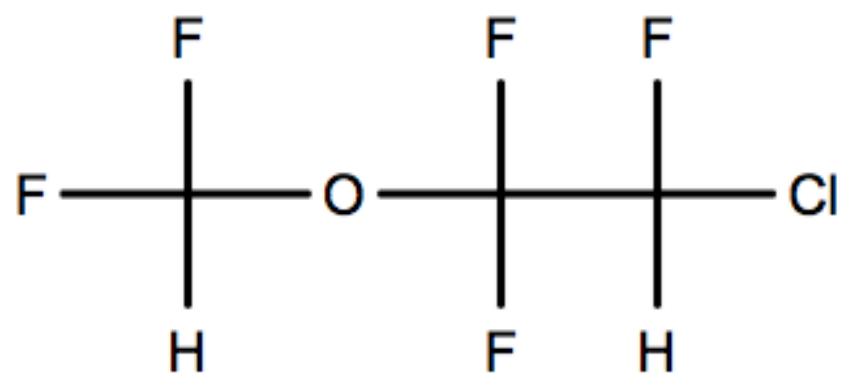

Figure 1 

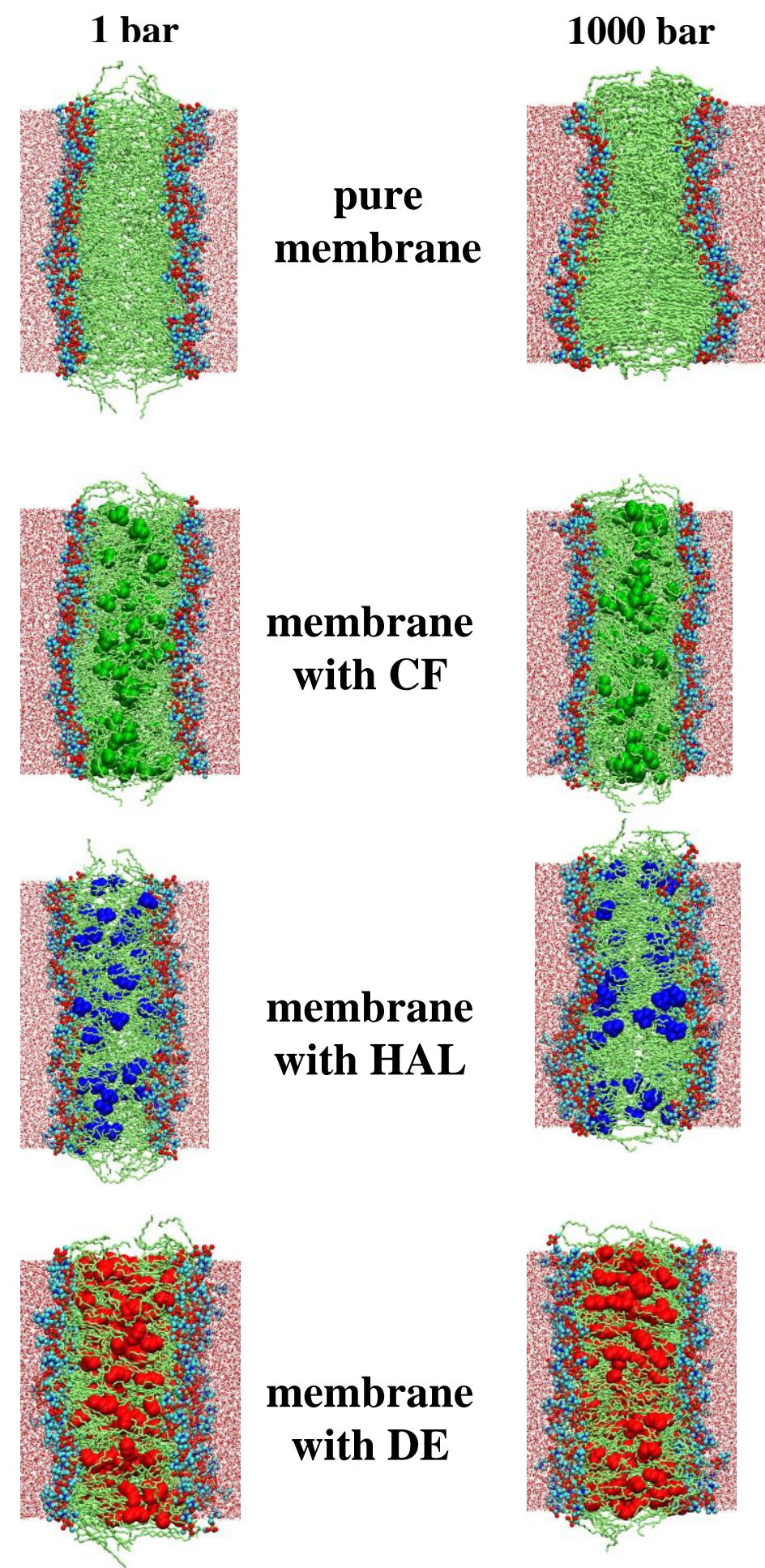

membrane with DE

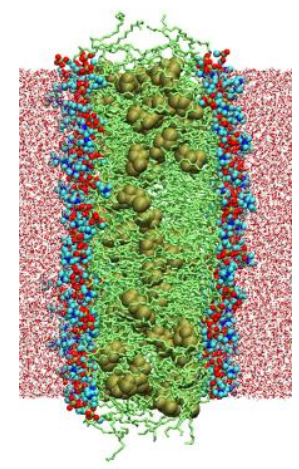

membrane with ENF
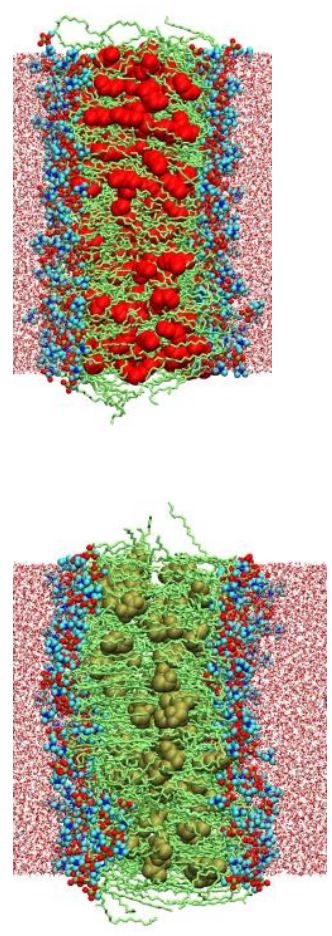

Figure 2 


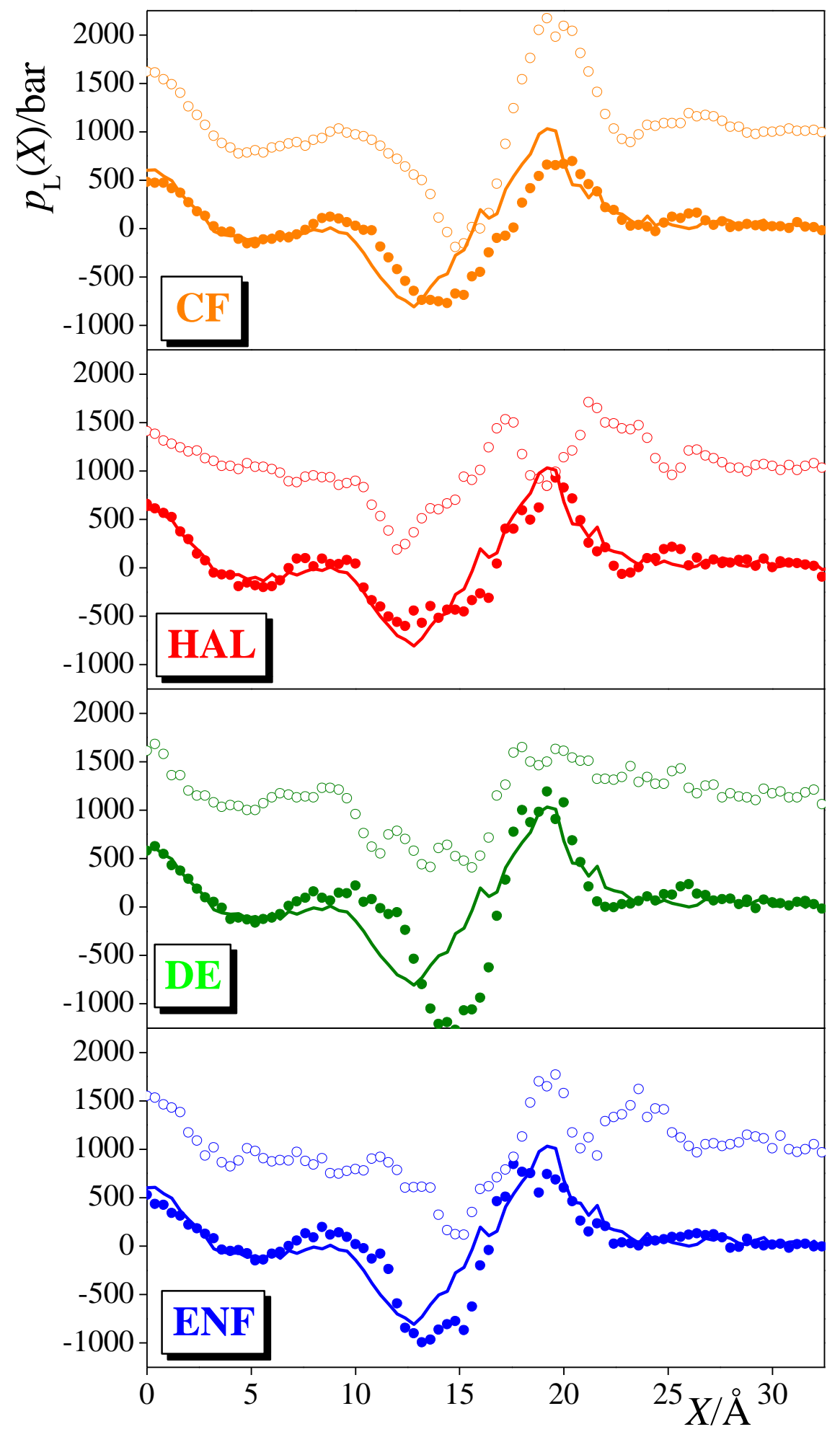

Figure 3 


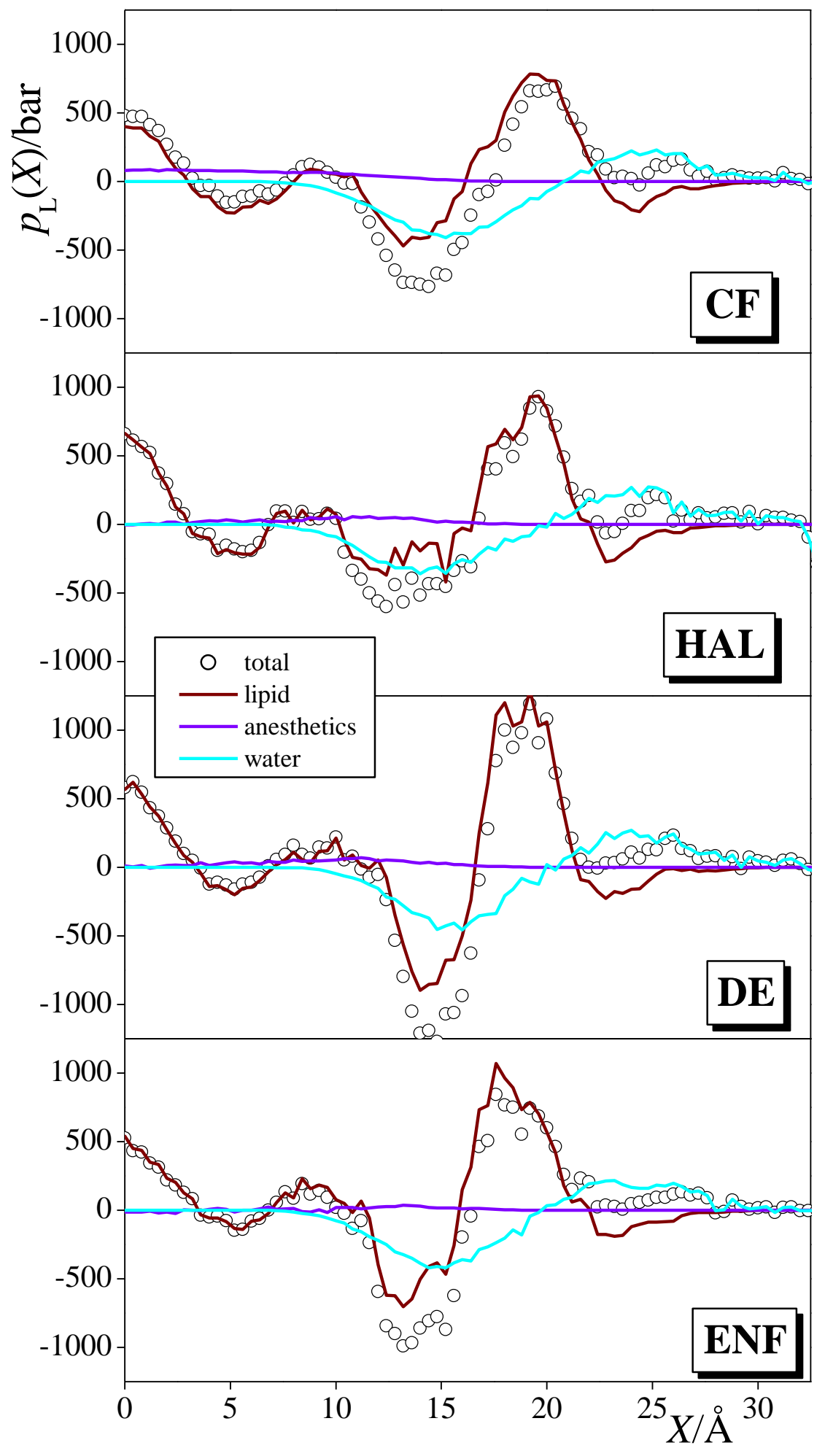

Figure 4 


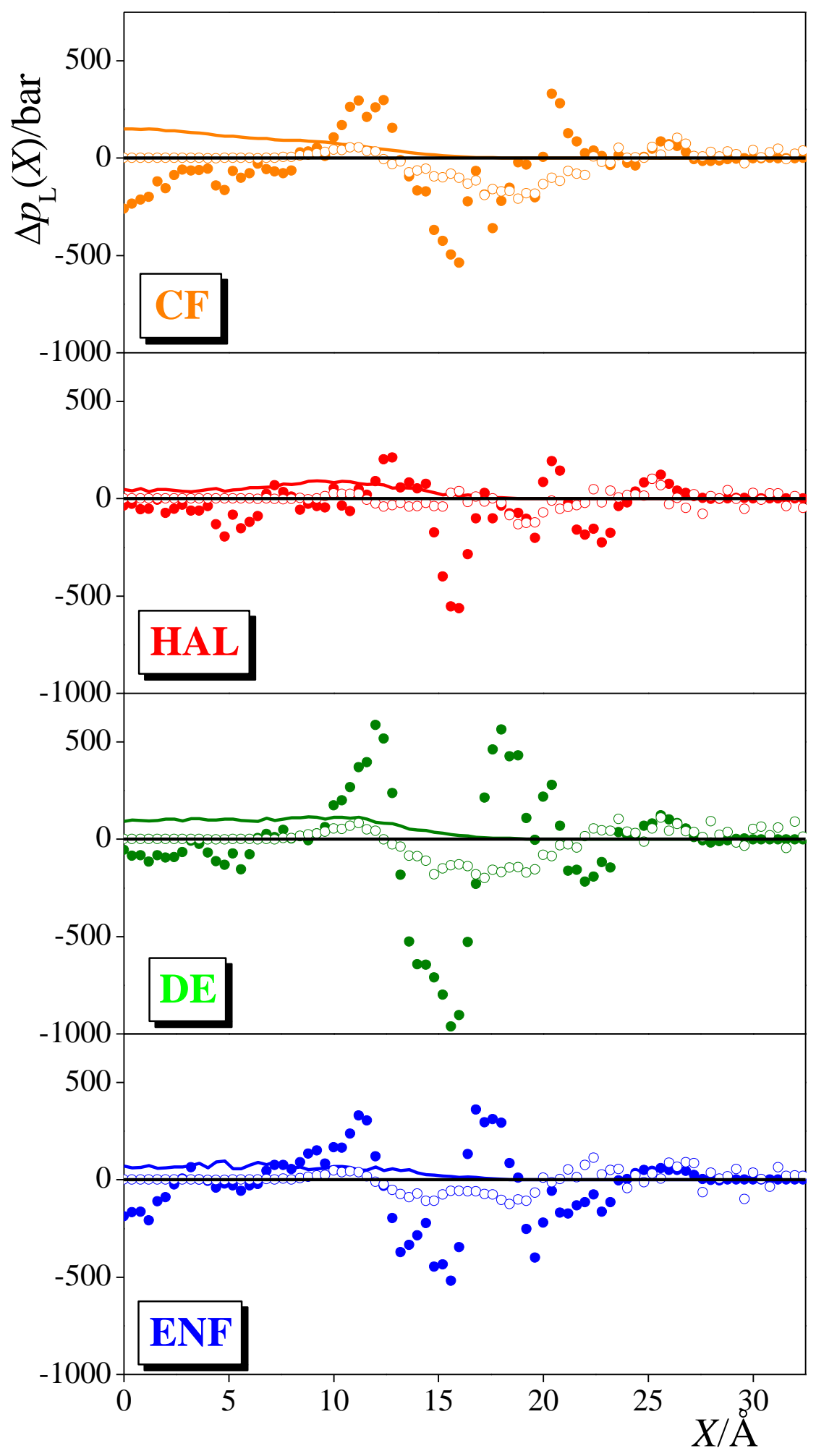

Figure 5 


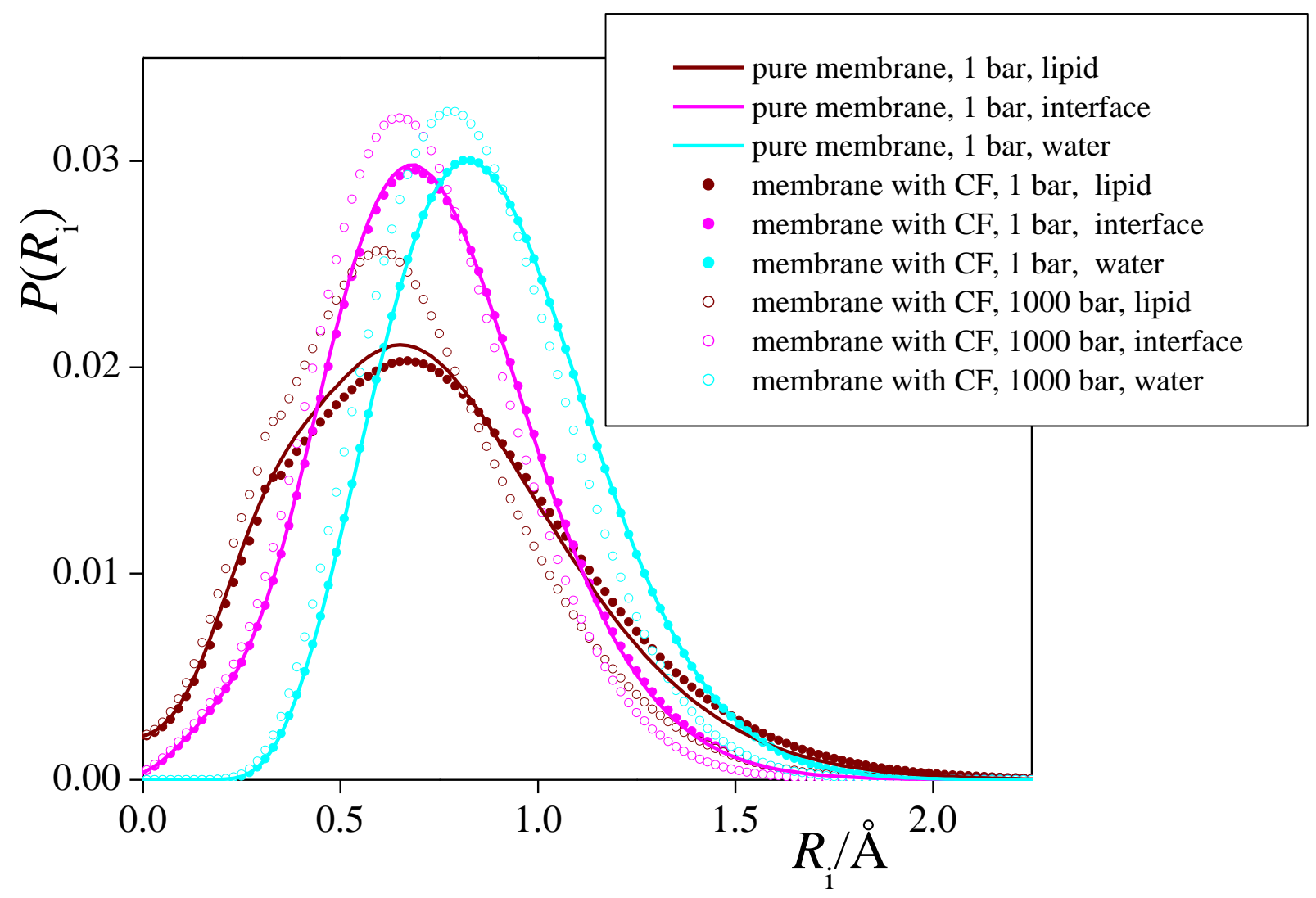

Figure 6 


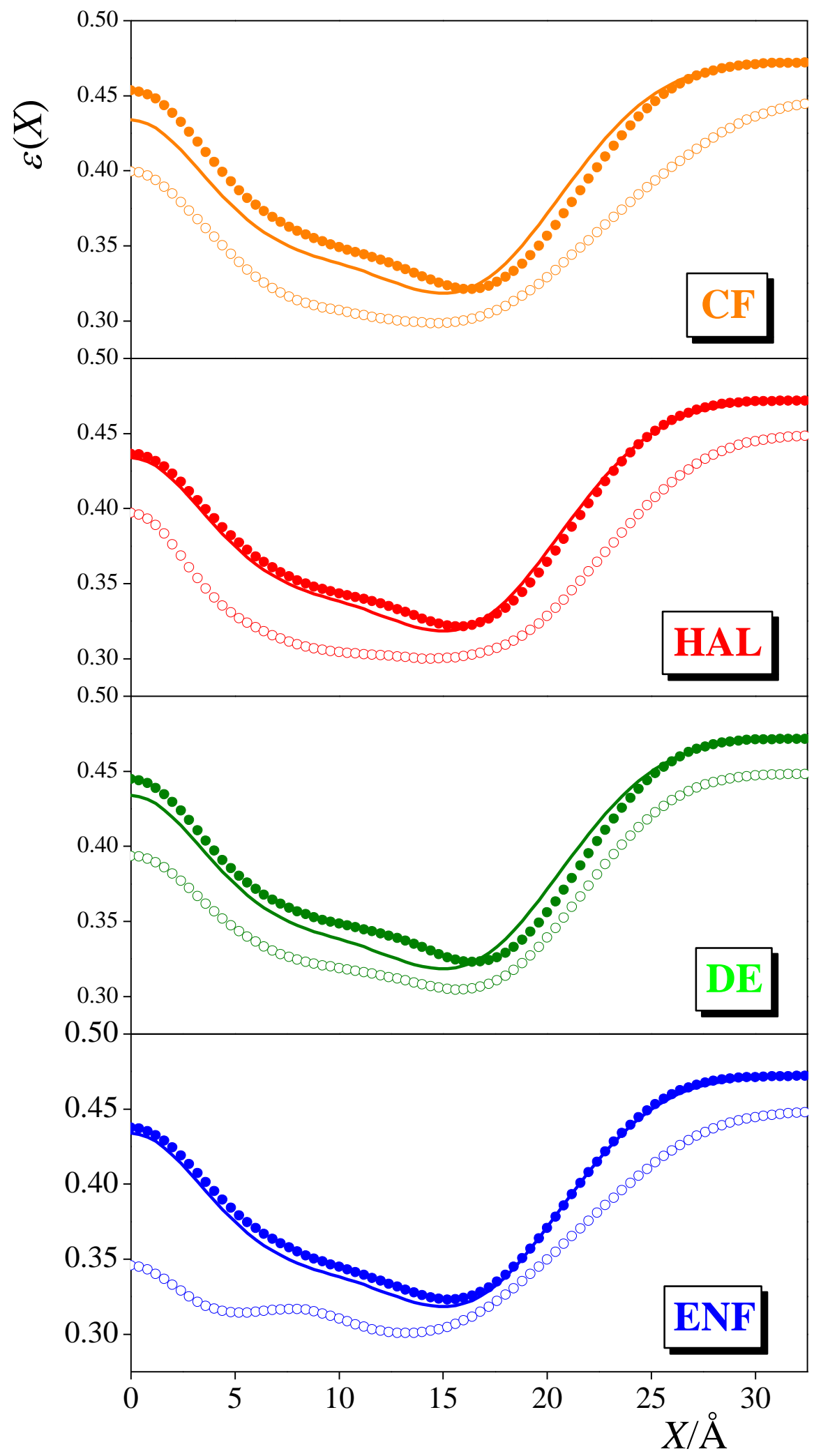

Figure 7 
TOC IMAGE

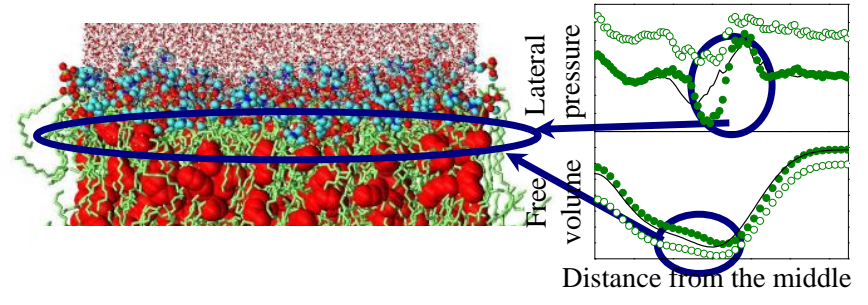

